\title{
Re-Globalisierung. Überlegungen zu einem Epochenbegriff für die Gegenwart
}

\author{
Roland Benedikter
}

Angenommen: 22. November 2021 / Online publiziert: 11. Januar 2022

(C) The Author(s), under exclusive licence to Springer Fachmedien Wiesbaden GmbH, ein Teil von Springer Nature 2022

Zusammenfassung De-Internationalisierung und Re-Nationalisierung sind in Medien und Wissenschaft in ähnlichem Maße zu Schlagworten der Gegenwart geworden wie der Rückzug der Demokratie und die Krise internationaler Organisationen. All diese Unterbegriffe beschreiben eine Transformation des Globalisierungsmusters, das die Welt seit Beginn der 1990er-Jahre kannte. Der Begriff der Re-Globalisierung erfasst, wie die Globalisierung derzeit durch eine Vielzahl von Verschiebungen, Einflüssen, Ereignissen und Kräften neu konfiguriert wird.

Schlüsselwörter Re-Globalisierung · De-Globalisierung · DeInternationalisierung $\cdot$ Re-Nationalisierung $\cdot$ Glokalisierung

\section{Re-Globalization. Reflections on an Era-Defining Term}

Abstract De-internationalization and re-nationalization have become buzzwords in media and academia just like the retreat of democracy and the crisis of international organizations. These concepts describe the transformation of both a specific idea and practice of globalization which the world had known since the early 1990s. The term re-globalization captures how globalization is currently being reconfigured by a variety of shifts, influences, events, and forces.

Keywords Re-Globalization · De-Globalization · De-Internationalization · ReNationalization · Glocalization

Dr. Roland Benedikter $(\bowtie)$

Center for Advanced Studies - Eurac Research, Viale Druso / Drususallee 1, 39100 Bozen, Italien

E-Mail: roland.benedikter@eurac.edu 
Der Autor ist Co-Leiter des Center for Advanced Studies von Eurac Research Bozen, Italien, Forschungsprofessor für multidisziplinäre Politikanalyse in residence am Willy-Brandt-Zentrum der Universität Breslau, Polen, und Mitglied des Zukunftskreises des Bundesministeriums für Bildung und Forschung Berlin.

\section{Einleitung}

Re-Globalisierung ist ein Begriff, der beschreibt, wie die Globalisierung derzeit durch eine Vielzahl von Verschiebungen, Einflüssen, Ereignissen und Kräften neu konfiguriert wird. De-Internationalisierung und Re-Nationalisierung sind in Medien und Wissenschaft in ähnlichem Maße zu Schlagworten der Gegenwart geworden wie der Rückzug der Demokratie und die Krise internationaler Organisationen. All diese Unterbegriffe (und ihre Begriffsfelder) beschreiben einen Übergang oder gar eine Transformation des Globalisierungsmusters, das die Welt seit Beginn der 1990erJahre kannte. In der Tat hat seit Beginn des 21. Jahrhunderts eine lange Reihe von Veränderungen und Ereignissen die Globalisierung beeinflusst und ihre Grundlagen langsam, aber stetig verändert.

Das Jahrhundert begann mit dem 11. September 2001. Er sandte Schockwellen der Polarisierung und Radikalisierung in die westliche Zivilisation, die noch immer nicht vollständig erfasst wurden. Hinzu kam die kontinuierliche Verschärfung der Umwelt- und Klimakrisen. Drittens entstand eine Technologiekrise, einschließlich der Beeinflussung von Wahlen und des wachsenden Einflusses der sozialen Medien auf die Demokratie, was den öffentlichen Diskurs zunehmend beeinflusste. Es folgten die Finanz- und Wirtschaftskrise 2007-2008; die europäische Schuldenkrise; der Arabische Frühling 2010-2011; die Migrations- und Flüchtlingskrisen seit 2014-2015; die Krise des EU-Einigungsprojekts, die 2016 im Brexit gipfelte; und die US-Präsidentschaft von Donald Trump 2017-2021 mit ihrem stark antiinternationalistischen Vorstoß. Als eines der jüngsten Ereignisse in einer Reihe, die bekannte Modalitäten, Mechanismen und Überzeugungen untergraben, hat sich die Coronavirus- (oder Covid-19-)Krise seit Ende 2019 als ein weiterer Schlag gegen die Praxis ,intensiver“ Globalisierung erwiesen, wenn nicht gar gegen Grundideen der Globalisierung und ihre (oft widersprüchlichen oder sogar gegensätzlichen) Ideologien des „Globalismus“ an sich (Steger 2001). Der durch Covid-19 verursachte Bruch untergrub den Glauben an ausgeprägte internationale Arbeitsteilung und schadete dem Ansehen jener globalen Institutionen und Organisationen, die von den westlichen Demokratien nach dem Zweiten Weltkrieg geschaffen worden waren. Nationale Perspektiven und eine allgemeine Tendenz zur „Autonomisierung“ und Selbstversorgung traten wieder stärker in den Mittelpunkt.

Darüber hinaus wurde die Globalisierung in den letzten Jahren durch die systematische Schaffung paralleler Ordnungen durch aufstrebende illiberale Akteure wie China und Bündnisse wie die Shanghaier Organisation für Zusammenarbeit verändert, was im Gesamtbild nicht unterschätzt werden darf. Seitdem gibt es nicht mehr nur eine Globalisierung, wie es im unbewussten Inhalt des Begriffs mitschwingt, sondern viele Globalisierungen nebeneinander. Diese konkurrieren miteinander, liegen zum Teil miteinander im Kampf und weisen trotz enger infrastruktureller, finan- 
zieller, ökonomischer oder technologischer Verzahnung in vielen Aspekten kaum ideologische Berührungspunkte auf. Ein Beispiel dafür, wie in ein und demselben Phänomen viele Globalisierungen, konkurrierende politische Systeme und gegensätzliche Ideologien koexistieren, während sich die Sichtweisen der beteiligten Akteure auf das damit verbundene Globalisierungsgeschehen stark unterscheiden, ist Chinas „Neue Seidenstraße“-Projekt nach Europa. Symptomatisch für den ReGlobalisierungsprozess wurde dieses größte Infrastrukturprojekt der Geschichte von einem geschlossenen und autoritären Regime mit Ziel demokratische offene Gesellschaften ohne vorherige Absprache oder Abkommen begonnen. Dabei sind China und die EU, unabhängig von ihrer Mitgliedschaft in internationalen und globalen Organisationen, ,systemische Rivalen“ in ein und demselben Prozess, wie es seit 2019 EU, NATO, USA und G7 erklärten.

All dies sind Anzeichen dafür, dass die Globalisierung ihr Gesicht verändert. Gleichzeitig fragen sich viele mit wohlbegründeten Argumenten, ob die gegenwärtige Phase des Internationalismus, welche die Dinge in Richtung einer multipolareren, vielschichtigeren und in vielerlei Hinsicht mehrdeutigeren globalen Struktur neu ordnet, überhaupt als „Re-Globalisierung“ bezeichnet werden sollte. Dies könnte $\mathrm{zu}$ reform- und erneuerungsorientiert für eine historische Phase klingen, die in ihrem Charakter eher chaotisch und in vielen ihrer Bestrebungen und Wirkungen eher regressiv charakterisiert zu sein scheint. Im vorliegenden Beitrag werden einige grundsätzliche Vor- und Nachteile des Begriffs Re-Globalisierung und seine mögliche Verwendung als interdisziplinärer Integrationsbegriff gegenwärtigen Wandels erörtert. Re-Globalisierung ist ein Begriff mit einem gewissen umfassenden Anspruch, der in mehrerlei Hinsicht selbstkritisch überprüft werden muss. Er weist zudem klare Geltungsgrenzen auf.

\section{Re-Globalisierung, oder: Indikatoren für eine Epoche von Übergang, Unsicherheit und Wandel}

Re-Globalisierung ist - am Beginn jeder Grundsatzreflexion - die Wahrnehmung eines zunächst diffusen Musters der Veränderung, das sich seit einigen Jahren durch alle disziplinären Felder der Weltgesellschaft zu ziehen scheint. ,Weltordnung ohne Hüter": So kennzeichnete der deutsche Politikwissenschaftler Herfried Münkler im Mai 2020 die aktuelle historische Phase aus politischer Sicht (El-Safadi 2020). Er meinte damit die Tendenz zum Abhandenkommen einer klaren Leitstruktur jenes Internationalismus, den der demokratische Westen nach dem zweiten Weltkrieg mittels einer liberalen globalen Zivilordnung und entsprechender Institutionen errichtet hatte. Wirtschaftswissenschaftler*innen sprechen seit einigen Jahren aus ökonomischer Sicht von der Krise und dem möglichen Ende des globalen (wenn vielleicht auch nicht flächendeckend des regionalen) Neoliberalismus. Dies wurde auch mit ausgelöst durch die De-Globalisierungstrends der Coronavirus-Pandemie, die zu einer Bewegung des ,neuen Selbermachens“ geführt hat. Die für das Selbstverständnis der Globalisierung der 1990er- und 2000er-Jahre grundlegende transnationale Zirkulation von Kapital und Arbeitsteilung ist seit Jahren wachsender Grundsatzkritik ausgesetzt, weil sie Steuerflucht begünstigt und Ungleichheit gefördert hat. Sie hat 
gemeinsam mit anderen Faktoren dazu beigetragen, dass nach und nach auch immer größere Personenmengen - und mit ihnen Kulturen, zivilisatorische Paradigmen und Religionen - in Bewegung und Vermischung (oft ohne Integration) gerieten. Drittens sprechen Kulturwissenschaftler*innen bereits seit den 2010er-Jahren aus kultureller Sicht von einer Phase der „Post-Postmoderne“ und „Meta-Moderne“. Diese versucht, ins Jenseits bisheriger Dichotomien von Links- und Rechtsinterpretationen von Globalisierung und Gesellschaft auszugreifen. Sie konstatieren: Die aktuelle Ära ist gerade in jenen entwickelten Gesellschaften, die die Globalisierung lanciert und gelenkt haben, dadurch gekennzeichnet, dass deren Bürger*innen durch die komplex ineinandergreifenden Wirkungen ernüchtert sind. Unter diesen Wirkungen ist die Kombination von Delokalisierung mit immer stärkeren Individualisierungswirkungen „sozialer Technologien“, die bis zu einem „kosmopolitischen Solipsismus“ reichen. Als Reaktion darauf tendieren diese Gesellschaften - zu denen auch die europäischen gehören - dazu, den säkularen Dekonstruktivismus, der sie selbst-, gemeinschafts- und bewusstseinskritisch seit den 1970er-Jahren gekennzeichnet hat, zwar nicht zu verlassen, aber doch aktiv zu überschreiten. Sie suchen statt der von der Postmoderne geforderten Zertrümmerung umfassender Narrative (bei gleichzeitiger Auflösung „großer Erzählungen“ in Einzelgeschichten und individuellen Konstrukten) nach neuen Narrativen, welche Realismus und Idealismus vereinen können. Dies, ohne wie die Moderne (instrumenteller Realismus) und Postmoderne (negativer Idealismus) eine der beiden Seiten zu bevorzugen. Das hat damit zu tun, dass sich aus Sicht der Globalisierungs-Ernüchterten herausgestellt hat, dass narrativlose Gesellschaften der Globalisierung nicht gewachsen sind. Denn sie können von deren Wirkungen - gerade auch im imaginären Bereich - fast widerstandslos penetriert werden. Für ein balancierteres - und damit resilienteres - Paradigma ist, so die Stimmung dieses westlichen Zeitgeists, in Bezug auf die zwei typologischen Leitdiskurse der Moderne eine Abwendung vom idealistischen „Sowohl-als-Auch“ Hegels und eine Hinwendung zu einem realistischeren (nun jedoch als „,bodenständige“ Vernunft oder „,common sense“ interpretierten) „Entweder-Oder“ Kierkegaards notwendig (Henriques 2020; Nealon 2012; Turner 2015).

„Künstler-Intellektuelle“ wie der australisch-britische Musiker und Schriftsteller Nick Cave sprechen in diesem paradigmenverändernden Sinn von der Re-Globalisierung als einer Ära der „Notwendigkeit, die Welt [geistig] zu rehabilitieren“ (Richards 2020). Aus Caves Sicht ist die „Tiefe“ der politischen, ökonomischen und kulturellen wie der damit zusammenhängenden mentalen Veränderung so umfassend, dass

wir in einer beängstigenden und zutiefst unsicheren Zeit leben. Und obwohl es da draußen wahnsinnige und zynische Stimmen gibt, die durch die sozialen Medien - diese verrückten Motoren der Empörung - ermutigt und verstärkt werden, repräsentieren sie nicht die Stimmen der Vielen. Meine Erfahrungen mit den Menschen in unserer Zeit sind überwältigend positiv. Ich denke, dass die meisten von uns verstehen, dass wir an einem Strang ziehen und mit Höflichkeit, Großzügigkeit und Freundlichkeit handeln müssen, um diesen besonderen Moment zu überstehen. Wir haben eine monumentale Aufgabe vor uns, die enorme Energiereserven erfordern wird: wir müssen die Welt rehabilitieren, [eine Welt wiederherstellen]. Natürlich gibt es vieles, das verändert und 
in Ordnung gebracht werden muss, und natürlich ist die Menschheit komplex, widersprüchlich und voller Fehler. Aber in diesem Augenblick, in dem unsere Existenz auf dem Spiel steht, müssen wir nicht nur in gutem Glauben und gegenseitigem Trost zusammenkommen, sondern auch in einem Geist der Kreativität und des Erfindungsreichtums. Unsere Existenz hängt davon ab, dass wir das Beste unserer selbst anbieten. Negativität, Zynismus und Ressentiments werden nicht ausreichen. Wir müssen uns gegenseitig lieben oder sterben (Richards 2020).

Diese Worte verweisen auf eine Emotionalisierung, die mit der Re-Globalisierung einhergeht und Teil ihrer historischen Symptomatologie ist. Kritiker*innen sehen im Kern der negativen Wirkungen von Globalisierung vor allem die Effekte der instrumentellen Allianz zwischen neoliberalem Realismus und linksliberal-kosmopolitischem Idealismus, die die „klassische“ Globalisierung zwischen 1989 und 2015 geprägt hat. Das war eine unheilige Allianz in (scheinbar) beiderseitigem Interesse, bei der eine Seite die andere für die eigenen Absichten zu vereinnahmen oder zumindest zu gebrauchen suchte. Das resultierende neoliberal-kosmopolitische Denkmodell wurde meist mehr oder weniger oberflächlich angewandt, um Widersprüche zwischen den beiden ungleichen Partnern zu überspielen. Oft wurde der neoliberale Kosmopolitismus, der mindestens genauso sehr ein kosmopolitischer Neoliberalismus war, mit Hilfe von Wissenschaft und Medien zur Mainstream-Propaganda ausgeformt. Der Globalisierungs-Slogan sollte trotz früher Warnzeichen eine gewisse beruhigende Mitte herstellen, um die neoliberal-kosmopolitische Interpretation von Globalisierung als Leitmentalität zumindest in den offenen Gesellschaften des Westens zu verankern. Dies wird seit einigen Jahren in Frage gestellt und von vielen als Illusion einer bestimmten historischen Phase kritisiert - einschließlich der damit verbundenen Selbstinterpretationen des Globalismus.

„Die Welt zu rehabilitieren“ heißt aus kritischer Sicht deshalb zunächst, die Widersprüche dieses bisher leitenden Denkmodells der Globalisierung offenzulegen. Das wird in einer Phase der Verschiebungen und Umbrüche leichter, in der sowohl Realismus als auch Idealismus nicht mehr das bleiben können, was sie bislang vorgaben zu sein. Denn beide werden von globalisierungsbedingten Krisen herausgefordert, für die es bis auf weiteres offenbar keine eindeutige oder abschließende Lösung gibt.

\section{Re-Globalisierungsrhetorik der Vereinten Nationen}

UN-Generalsekretär António Guterres formulierte diese Gesamtsituation von Krise und Übergang, die die Gegenwart kennzeichnet und einen kulturellen Mentalitätswandel des Westens antreibt, anlässlich des 75. Jahrestags der Vereinten Nationen im Juni 2020 als die „Krise des Multilateralismus“. Dabei interpretierte er Re-Globalisierung als „reinvention“, also „Neuerfindung“ (nicht: Wiederherstellung!) von Weltgemeinschaft: 
UN-Generalsekretär Guterres hat sich zum 75. Jahrestag der Gründung der Vereinten Nationen für eine Neuerfindung des Multilateralismus ausgesprochen. Der derzeitigen Zusammenarbeit der internationalen Staatengemeinschaft fehle es an „Bedeutung, Ehrgeiz und Biss“, sagte Guterres. Es sei schwierig, ohne die aktive Teilnahme der Weltmächte eine bedeutungsvolle Veränderung der Mechanismen globaler Führung zu erreichen, räumte Guterres ein. Deren Beziehungen untereinander ,waren noch nie dysfunktionaler“. Er hoffe, dass die Coronavirus-Krise ein Weckruf für einen neuen Multilateralismus sei, so der UN-Generalsekretär (Austrian Press Agency 2020b).

Guterres’ Betonung lag ausdrücklich auf einem „,neuen“ Multilateralismus - was sowohl die umstürzende wie innovative Funktion der Re-Globalisierungsphase betonte. In anderem Zusammenhang wurde Guterres hinsichtlich Zustand von Welt und Krise der Globalisierung allerdings noch deutlicher, ja offen apokalyptisch und sogar beschwörend-protoreligiös in seiner Rhetorik. In zwei wegweisenden „GroßesBild“-Reden von 2020 - zu den Jahreszielen der UN im Januar und zum 75-JahrJubiläum der UN im Juni desselben Jahres - äußerte Guterres unter anderem:

Ich sehe [mit Blick auf die gegenwärtige Welt] ,vier Reiter der Apokalypse“ in unserer Mitte: vier drohende Gefahren, die den Fortschritt des 21. Jahrhunderts gefährden und die Möglichkeiten des 21. Jahrhunderts aufs Spiel setzen. Der erste Reiter kommt in Form der stärksten globalen geostrategischen Spannungen, die wir seit Jahren erlebt haben. Verheerende Konflikte verursachen weit verbreitetes Elend. Terroristische Anschläge fordern gnadenlosen Tribut. Die nukleare Bedrohung nimmt zu. Mehr Menschen sind durch Krieg und Verfolgung aus ihrer Heimat vertrieben worden als jemals zuvor seit dem Zweiten Weltkrieg. Die Spannungen in den Bereichen Handel und Technologie bleiben ungelöst. Die Gefahr eines großen Bruchs ist real. Zweitens stehen wir vor einer existenziellen Klimakrise. Der Temperaturanstieg lässt Rekorde schmelzen. Das vergangene Jahrzehnt war das wärmste seit Beginn der Aufzeichnungen. Nach Angaben von Wissenschaftlern steigen die Temperaturen in den Ozeanen mit der Geschwindigkeit von fünf Hiroshima-Bomben pro Sekunde. Eine Million Arten sind in naher Zukunft vom Aussterben bedroht. Unser Planet verbrennt. Wie wir auf der [Weltklimakonferenz] COP25 [2.-13. Dezember 2019] gesehen haben, tüfteln zu viele Entscheidungsträger weiterhin herum, ohne zu handeln. Unsere Welt nähert sich dem Punkt, an dem es kein Zurück mehr gibt. Und der dritte apokalyptische Reiter ist ein tiefes und wachsendes globales Misstrauen. Beunruhigung und Unzufriedenheit erschüttern die Gesellschaften von Nord bis Süd. Jede Situation ist einzigartig, aber überall füllt Frustration die Straßen. Immer mehr Menschen sind überzeugt, dass die Globalisierung für sie nicht funktioniert (Guterres 2020).

Der UN-Generalsekretär erläuterte diese Unruhe in Bezug auf die Globalisierungsmuster im Weiteren ausführlicher und stellte fest:

Zwei von drei Menschen leben in Ländern, in denen die Ungleichheit zugenommen hat. Das Vertrauen in das politische Establishment sinkt. Junge Menschen 
erheben sich. Frauen fordern zu Recht Gleichberechtigung und Freiheit von Gewalt und Diskriminierung. Gleichzeitig machen sich Ängste und Befürchtungen breit. Die Feindseligkeit gegenüber Flüchtlingen und Migranten nimmt zu. Der Hass wächst (Guterres 2020).

Dies veranlasste Guterres schließlich dazu, als „,vierten Reiter“ der Apokalypse den techno-transformativen Zustand zu erwähnen, der ebenfalls ein entscheidender Bestandteil der Re-Globalisierung ist:

Die vierte Bedrohung ist die dunkle Seite der digitalen Welt. Der technologische Fortschritt schreitet schneller voran, als wir in der Lage sind, auf ihn zu reagieren oder ihn überhaupt zu verstehen. Trotz enormer Vorteile werden die neuen Technologien missbraucht, um Verbrechen zu begehen, Hass zu schüren, Informationen zu fälschen, Menschen zu unterdrücken und auszubeuten und in die Privatsphäre einzudringen. Wir sind auf die tiefgreifenden Auswirkungen der vierten industriellen Revolution auf den Arbeitsmarkt und die Struktur der Gesellschaft nicht vorbereitet. Künstliche Intelligenz schafft atemberaubende Kapazitäten und alarmierende Möglichkeiten. Tödliche autonome Waffen, Maschinen, die in der Lage sind, ohne menschliches Urteil und ohne Verantwortung zu töten, bringen uns auf ein moralisch und politisch inakzeptables Terrain (Guterres 2020).

Nach Ansicht des UN-Generalsekretärs verändern diese ,vier Reiter der Apokalypse" die bisherige Globalisierung und ihre Ordnungsparameter in Richtung einer ebenso umfassenden wie tiefgreifenden Re-Globalisierung. Seiner Ansicht nach ist Re-Globalisierung jedoch im Wesentlichen eine Gratwanderung auf des Messers Schneide - ja sogar die Gratwanderung unserer Zeit, bei der sich vieles entscheidet:

Diese vier Reiter: epische geopolitische Spannungen, die Klimakrise, globales Misstrauen und die Schattenseiten der Technologie können jeden Aspekt unserer gemeinsamen Zukunft gefährden. Deshalb reicht es nicht aus, den 75 . Jahrestag [der Vereinten Nationen] mit schönen Reden zu begehen. Wir müssen diese vier Herausforderungen des 21. Jahrhunderts mit vier Lösungen des 21. Jahrhunderts angehen. Wir sehen überall auf der Welt gordische Knoten von der Golfregion bis zum israelisch-palästinensischen Konflikt, von der Sahelzone und dem Tschadsee bis Venezuela. Resolutionen des Sicherheitsrates werden ignoriert. Einmischung von außen schürt Brände. Und wir laufen Gefahr, Pfeiler der internationalen Abrüstungs- und Rüstungskontrollarchitektur zu verlieren, ohne dass es tragfähige Alternativen gibt. Prävention muss unser gesamtes Handeln bestimmen, wenn wir uns für ein Friedenskontinuum engagieren. Wir müssen unsere Vermittlungskapazitäten und Instrumente zur Aufrechterhaltung des Friedens stärken, die allein zu langfristiger Entwicklung führen. Der 20. Jahrestag der Resolution 1325 des Sicherheitsrates zu Frauen, Frieden und Sicherheit ist auch die Gelegenheit, Worten Taten folgen zu lassen. Und wir müssen uns auf die Wurzeln von Krisen und Umbrüchen konzentrieren und die Triebkräfte von Gewalt und Extremismus bekämpfen: von Ausgrenzung bis zu wirtschaftlicher Verzweiflung, von gewalttätiger Frauen- 
feindlichkeit bis zu Regierungsversagen. Denn alles ist miteinander verbunden (Guterres 2020).

Dann kam Guterres zu seinen Lösungsvorschlägen im Hinblick auf den „dritten Reiter":

Um den dritten Reiter, das weltweite Misstrauen zu besiegen, müssen wir eine faire Globalisierung aufbauen. Wir haben einen Plan. Er heißt Agenda 2030 für nachhaltige Entwicklung. Wir sehen konkrete Fortschritte, von der Senkung der Kindersterblichkeit bis zur Ausweitung der Bildung, von der Verbesserung des Zugangs zur Familienplanung bis zur Ausweitung des Internetzugangs. Wir rufen ein Aktionsjahrzehnt ins Leben, um die Ziele für nachhaltige Entwicklung bis $2030 \mathrm{zu}$ erreichen. Dieses Aktionsjahrzehnt [2020-2030] ist von zentraler Bedeutung für die Verwirklichung einer fairen Globalisierung, die Ankurbelung des Wirtschaftswachstums und die Verhütung von Konflikten. Während des Aktionsjahrzehnts müssen wir in die Beseitigung der Armut, sozialen Schutz, Gesundheit und Bekämpfung von Pandemien, Bildung, Energie, Wasserversorgung und Abwasserentsorgung, nachhaltigen Verkehr und Infrastruktur sowie in universalen Internetzugang investieren. Wir müssen die Regierungsführung verbessern, illegale Finanzströme bekämpfen, die Korruption ausrotten und wirksame, vernünftige und gerechte Steuersysteme entwickeln. Wir müssen Volkswirtschaften für die Zukunft aufbauen und menschenwürdige Arbeit für alle sicherstellen, insbesondere für junge Menschen. Und wir müssen einen besonderen Schwerpunkt auf Frauen und Mädchen legen, weil dies uns allen zugute kommt (Guterres 2020).

\section{Abschließend widmete sich Guterres noch der Lösungsfindung zum „,vierten Rei-} ter":

Schließlich: Um die Schattenseiten der digitalen Welt zu bekämpfen, müssen wir Technologien für positive Veränderungen nutzen. Wir müssen die Bildungssysteme neu gestalten. Es geht nicht nur darum, zu lernen, sondern zu lernen, wie man lernt, ein Leben lang. Wir brauchen innovativere Ansätze für soziale Sicherheitsnetze. Wir müssen das Konzept der Arbeit und das lebenslange Gleichgewicht zwischen Arbeit, Freizeit und anderen Aktivitäten überdenken. Wir müssen Ordnung in den Wilden Westen des Cyberspace bringen. Im Jahr 2021 wird die Cyberkriminalität 6 Billionen US-\$ kosten. Der Cyberspace selbst läuft Gefahr, sich in zwei Teile zu spalten. Wir müssen der digitalen Fragmentierung entgegenwirken, indem wir die globale digitale Zusammenarbeit fördern (Guterres 2020). ${ }^{1}$

Wie immer man diese Analysen auch im Einzelnen ausinterpretiert: Re-Globalisierung scheint sowohl bei Experten wie auch beim Generalsekretär der wichtigsten globalen Organisation die verbindende Begriffsbrücke zwischen Diagnoseversuchen auf politischen, ökonomischem und kulturellem Feld zu sein. Sektorale Krisen mö-

\footnotetext{
${ }^{1}$ Die für 2021 erwarteten Kosten durch Cyberkriminalität entsprechen damit etwa $7 \%$ des geschätzten globalen BIP von etwa 87 Bio. US-\$.
} 
gen diese Re-Globalisierungsphase heraufbeschworen haben; sie bleiben dabei aber nicht sektoral. Einzelereignisse und Krisen waren nicht Ursachen, sondern (oftmals untereinander vernetzte oder voneinander angestoßene) Auslöser und Verstärker von Prozessen, die innerhalb der neoliberal-kosmopolitischen Globalisierung mutmaßlich bereits seit einiger Zeit im Sinne unterschwelliger Fermentierungen von Leitparadigmen und -praktiken im Gang waren.

\section{Was ist Re-Globalisierung?}

Aus alledem folgen erstens einige inhaltliche und zweitens einige formale Implikationen für ein heuristisch-grundlegendes Verständnis von Re-Globalisierung.

Erstens inhaltlich. Ein erster möglicher Zugang ist ein empirischer und dabei naivrealistischer. Die aktuelle Phase der Globalisierung wäre in diesem Sinn als äußerer Vorgang zu verstehen. Das impliziert, in einem ersten Schritt die wichtigsten Entwicklungen und Themen der Re-Globalisierung zu identifizieren und zueinander in Beziehung zu setzen. Für Europäer*innen waren die zwei wichtigsten Themen am Beginn des Jahres 2020 laut transnationalen Umfragen Umwelt und Jobs. Im November 2019 rief das EU-Parlament den Klimanotstand aus. Die Umweltkrise und die Transformation der Arbeit zählen aus europäischer Sicht zu den wichtigsten Katalysatoren der Re-Globalisierung. Die Jugend demonstriert jeden Freitag für Natur und soziale Gerechtigkeit. Zwei große Themen, die viele Bürger*innen in offenen politischen Systemen beschäftigen, sind Ungleichheit und Unsicherheit. Aber auch zunehmende religiöse und kulturelle Vielfalt, der steigende Einfluss von Meinungsmedien, Populismus, der Aufstieg des Überwachungsstaates China und die Entwicklungen in Russland und Afrika sind Veränderungen, die sich auf die bisherige globale Ordnung auswirken.

Diese synchrone Vielfalt an Veränderungen kann verwirrend sein. In einer Zeit, in der Unsicherheit (uncertainty) zur neuen Normalität wird (Müller 2019), heißt es deshalb zumindest heuristisch das Ganze des Umbruchs ins Auge zu fassen. Obwohl das angesichts der gleichzeitigen Zunahme von Komplexität und Geschwindigkeit schwierig oder vielleicht sogar unmöglich ist, ist es nötig, einen approximativen Überbegriff für den Epochenwandel zu setzen, um die Synchronie verschiedener Veränderungen zu erfassen und die Charakteristik der Gegenwart zu kennzeichnen.

In diesem Sinn ergeben sich zweitens einige formale Implikationen, die den Gebrauch des Begriffs Re-Globalisierung betreffen. Das ist ein eher (de-)konstruktivistischer und reflexiver Zugang, der auf den empirischen und naiv-realistischen aufbaut. Der Sammelbegriff „Re-Globalisierung“ kennzeichnet - in experimenteller und inter- und transdisziplinärer Weise - einen Zeitraum „großen“ und „tiefen“ Umbruchs, der durch die Verflechtung der oben beschriebenen Einzelentwicklungen gekennzeichnet ist. Damit ist nicht notwendigerweise ein Paradigmenwandel gemeint, sondern eher die (zwischen politischen Systemen asymmetrische) Gleichzeitigkeit verschiedener Paradigmenveränderungen ungleicher Art und ungleichen Ausmaßes. Sie alle haben jedoch gemein, dass sie bisherige Selbstverständnisse sowie Mechanismen und Perspektiven von Globalisierung verändern und verschieben. 
Das Ergebnis ist eine Neukonstitution von „Globalisierung“ und ihres Verhältnisses zu den Ebenen des Nationalen, des Regionalen und des Lokalen.

Diese Neukonstitution wird inzwischen häufiger als vordem auch als „Glokalisierung" bezeichnet. Damit ist neben einer Kritik von Globalisierungswirkungen sowie einer Rücknahme von Globalisierungsbeteiligungsbereitschaft vor allem auch eine stärkere Kontextualisierung und damit Ausdifferenzierung von Globalisierung hinsichtlich konkreter Anforderungen und Perspektiven einzelner Territorien gemeint. Eine solche Kontextualisierung wurde von der „klassischen“ neoliberal-kosmopolitischen Globalisierung zugunsten einer Nivellierung vernachlässigt, die zu einem entscheidenden Grund für ihre negativen Effekte wurde. Wer annehmen würde, Re-Globalisierung könne einfach ein Bouncing-back zur angeblich „glücklichen“ Globalisierung der 1990er-Jahre bedeuten, würde den Resilienzgedanken restaurativ interpretieren und sich der Illusion hingeben, Geschichte könne rückgängig gemacht werden. Möglich scheint jedoch, dass Re-Globalisierung auf dem kleinsten (und passivsten) gemeinsamen Nenner ihrer Einzelstränge zumindest eine ,negative Wende“ implizieren kann, die bisherige liberale Errungenschaften zurückfährt oder im Rahmen zunehmend autoritärer globaler Gesamteinflüsse auf ihren Kern reduziert. Der Begriff „Re-Globalisierung“ kann, abhängig von Begriffsverwendung und dahinterstehender Ideologie, interpretatorisch jedenfalls gewisse Beziehungen sowohl zu den Begriffsfeldern der Resilienz wie der Regression aufbauen.

Nun hat der sozialwissenschaftliche Gebrauch von Begriffen die Eigenschaft, dass er mittels dieser Begriffe Realität zugleich sowohl (passiv) abbildet wie (selbst) aktiv herstellt. Beides ist unweigerlich und ausnahmslos derselbe Prozess, was Teile der Sozialwissenschaften gerade mit Bezug auf die Globalisierung lange zu wenig berücksichtigt haben, weil sie ihrer Abbildungs- und Reflexionsfunktion zu große Bedeutung zugemessen haben. Die Darstellung schafft jedoch den Gegenstand mit - und beeinflusst damit Globalisierung ebenso wie Re-Globalisierung. In diesem Sinn ist Re-Globalisierung als Abbildungs- und Reflexionsbegriff mit einem für die Zeit emblematischen Mehrfachcharakter ausgestattet. Das verleiht dem Begriff eine konstitutive Ambivalenz zwischen den drei Dimensionen anamnetisch, diagnostisch und therapeutisch. Der Begriff Re-Globalisierung kann nämlich auf alle drei Weisen verwendet werden: als Rekonstruktion, als Repräsentation und als aktive Strategie des Handelns. Diese drei Weisen werden bislang meist wenig reflektiert und nicht selten in ein und derselben Anwendung unsystematisch vermischt. Im Vordergrund steht bislang der nichtexplizite Gebrauch als diagnostischer Begriff. Die anamnetische Ebene, die eine differenzierte Phasen- und Epochengeschichte der Globalisierung seit dem Ende der 1980er-Jahre voraussetzt, ist erst im Entstehen (Steger et al. 2023). Dagegen ist die therapeutische Begriffsverwendung noch weitgehend unterentwickelt. Sie schließt sich bislang, wo sie versucht wird, an entsprechende Programme globaler Institutionen, darunter der Vereinten Nationen und ihrer Teilorganisationen sowie der Organisation für wirtschaftliche Zusammenarbeit und Entwicklung (OECD) an. Die Vermischung und gleichzeitige Ungleichgewichtung der drei Begriffsverwendungen ist bisher eine Schwäche des Re-Globalisierungsbegriffs, die seiner noch relativ jungen Gebrauchsgeschichte geschuldet ist. Alle drei Gebrauchsformen gleichermaßen weiterzuentwickeln, sie systematisch aneinander 
anzuschließen und mit einem unterscheidbaren empirischen Unterbau zu versehen, bleibt eine zentrale sozialwissenschaftliche Aufgabe.

\section{Eine hypothetische Epochenbeschreibung}

Zusammenfassend kann als Ausgangspunkt gelten: Re-Globalisierung ist eine derzeit im Ansatz begriffene, bis auf weiteres hypothetische Epochenbeschreibung uneinheitlichen Zuschnitts, ohne genaue Eingrenzung voraussehbarer Zeiträume und zunächst ohne ausreichende disziplinäre, methodische oder ,agitatorische“ (sozial handlungsorientierte beziehungsweise aktivistische) Zuspitzung. Der Re-Globalisierungsbegriff verfährt bislang weitgehend eklektisch und muss seine Fähigkeit zur Datenproduktion sowie zur praxisorientierten Policy-Information erst unter Beweis stellen. Bisher bezieht sich der Begriff eher auf zu erwartende Großszenarien als auf planbare Ereignisse oder Schritte. Bezogen auf integrative Zukunftsforschung und inklusive Zukünftebildung, wie sie die UNESCO in den letzten Jahren verstärkt im Rahmen ihrer Futures Literacy- Initiativen sowie der dazugehörigen Futures Literacy Labs ausgearbeitet hat, ist Re-Globalisierung bislang eher für Foresight (Vorausschau auf mögliche Zukünfte) und für Antizipation (Handeln in der Gegenwart aus der Vorausschau auf mögliche Zukünfte) brauchbar, weniger für operative Planung (Forecast; Programmierung von zeitlich umrissenen Perioden).

Insgesamt gilt vor diesem Hintergrund: Re-Globalisierung als Terminus bezeichnet eher ein Begriffsfeld (Plural) als einen spezifischen Leitbegriff (Singular). Bestehende Orientierungsansätze in diesem Begriffsfeld bleiben mehrheitlich deskriptiv bis spekulativ und erreichen die konzeptuelle und normative Ebene bislang kaum. Als Gegenstand inter- und transdisziplinärer sozialwissenschaftlicher Forschung besteht Re-Globalisierung im Kern im unsystematischen Umbruch bisheriger Ordnungsmuster, einschließlich der aus und in ihnen entwickelten Symmetrien, in den seit den 1990er-Jahren etablierten globalen und nationalen Institutionen, Politiktypologien und Beziehungshabitus. Als Programm schließt Re-Globalisierung grundlegende Reform-, Revisions- oder Erneuerungsversuche der ,klassischen“ Globalisierung seit den 1990er-Jahren ein, so wie sie unter anderen Guterres angeführt hat. Re-Globalisierung beschreibt also sowohl passive wie aktive, also sowohl dem „Geist der Geschichte“ geschuldete wie programmatisch organisierte Phänomene und Entwicklungen. Als Analyseinstrument umfasst das Begriffsfeld sowohl anamnetische, diagnostische wie therapeutische Ansätze und Versuche, die von sehr unterschiedlicher Qualität sind. Zweifellos ist „Re-Globalisierung“ dabei im Vergleich zur Diversität, Widersprüchlichkeit und „Veränderungstiefe“ gegenwärtiger Umbruchprozesse ein vereinfachendes und teilweise auch reduktionistisches Signalwort. Deshalb kann es auch keinen im strengen Sinn analytischen Charakter beanspruchen. Trotzdem gehört „Re-Globalisierung“ in das Spektrum der Zukunftsbildung, also der Futures Literacy, wie sie inzwischen auch größere Firmen wie Merck als Weiterentwicklung von Vorausschau- und Planungsprozessen sowie von traditionelleren Methoden wie Horizon Scanning, Datenprojektionsanalyse oder DelphiExpertenbefragungen propagieren (Miller 2019). Das entspricht der inhärenten Logik des Begriffs. Denn die der Re-Globalisierung impliziten Zukunftsprojektionen 
und deren (Vorlauf-)Prozesse werden sich bereits in der Gegenwart auswirken, je stärker die Gestaltungsebene mit dem Re-Globalisierungsthema zu arbeiten beginnt. Die zweifache Frage ist, welche Art ,andere“ oder gar „,neue“ Globalisierung „ReGlobalisierungsarbeit" hervorbringen kann, und wie lange das bis zum Erreichen von Ergebnissen dauern mag.

\section{Heuristische Datierungsansätze von Re-Globalisierung}

Vor dem Hintergrund der hier nur primordial angeführten Umrisse und Geltungsgrenzen kann Re-Globalisierung verschieden datiert werden. Weitgehende Übereinstimmung herrscht darin, dass sie mit Donald Trumps Wahl zum US-Präsidenten am 3. November 2016 zur politischen Tendenz und vom impliziten zum expliziten Prozess wurde. Der Schritt bestand in der Erhebung des „Antiglobalismus“ von rhetorischer Kritik an Fehlentwicklungen zum offiziellen politischen Programm der Regierung der wichtigsten Leitmacht und führenden Demokratie der Erde. Trumps offene Ablehnung „klassischer“, wenn nicht sogar jedweder Globalisierung überhaupt und sein Re-Nationalisierungsprogramm wurden für vier Jahre zum prägenden Muster globaler Veränderung. Sie prägten eine ideologische und geopolitische Perspektive, deren Sog auch Pro-Globalisierungsmächte wie die Europäische Union erfasste. Die EU unterlag zeitgleich ebenfalls dem Re-Nationalisierungstrend, wie bereits das Brexit-Referendum am 23. Juni desselben Jahres gezeigt hatte.

Es wäre aber ein historischer Fehler, Re-Globalisierung nur oder primär an großteils den negativen Wirkungen ,reifer“ Globalisierung geschuldeten - Umbrüchen innerhalb der demokratischen Leitmächte des Westens festzumachen. Re-Globalisierung entstand zumindest in Einzelaspekten der historischen Symptomatologie bereits mindestens seit Beginn der Ären dreier klassischer „starker Männer“: von Vladimir Putin (seit 1998/1999), Xi Jinping (seit 2012/2013) und Recep Tayyip Erdoğan (1994-1998 und seit 2003). Sie alle begannen Re-Nationalisierungsprogramme und stärkten nationale Souveränitätsideologien auf Kosten internationaler Gemeinschaftsformen. Sie bildeten Allianzen, um die liberale internationale Ordnung zu schwächen. Dabei bedienten sie sich einer charakteristischen Vermischung von Politik und Ökonomie mit Kultur und Religion, die sie strategisch aktiv handhabten. Sie fuhren Gleichheits-, Emanzipations- und Genderrechte zurück und beschworen - meist auch mithilfe des manipulativen Einsatzes neuer Technologien - einen neuen, meist hierarchisch-autoritär strukturierten gesellschaftlichen Unitarismus. Diese Politiken bewirkten eine Entdifferenzierung ihrer Gesellschaften. Diese Tendenz wurde für Aspekte der Re-Globalisierungsphase umso mehr kennzeichnend, je einflussreicher solche „,neounitarischen“ Politiken auf nationaler und internationaler Ebene wurden, und je stärker sie in globale Einrichtungen wie die Teilorganisationen der Vereinten Nationen (Sicherheitsrat, Menschenrechtsrat, Frauenrechtsrat, Weltgesundheitsorganisation, Welthandelsorganisation) hineinzuwirken in der Lage waren.

Eine frühe Signalwende für die „klassische“ Globalisierung war darüber hinaus wie erwähnt der 11. September 2001, der - von den USA ausgehend - die westlichen Gesellschaften weit stärker beeinflusste als heute noch oft angenommen (Mishra 
2020). Bereits die Amtszeit Barack Obamas (2009-2017) war im Rückblick ein Beitrag zur Lockerung des westlich-liberal begründeten Internationalismus, da Obama mit seiner strategisch-programmatischen Zuwendung zu Asien (Pivot to Asia) zugleich eine indirekte Abkehr von Europa verband (Benedikter 2012). Die seitdem in Gang gekommene Verschiebung von Gravitationszentren betrifft auch die Schwerpunktsetzung der Globalisierungsursprungsmythen Europas und des Westens. So wird inzwischen die Selbstbegründung bundesdeutscher liberaler Verfassung und Identität auf den Ereignissen der ersten Hälfte des 20. Jahrhunderts, insbesondere Weltkriege und Holocaust, gegenüber jener anderer Weltbereiche (Dekolonialisierung) relativiert oder gar indirekt in Frage gestellt (Mishra 2020). Ähnliches ist hinsichtlich der Wechselbeziehung zwischen US-amerikanischer Geschichte, Verfassung und Identität und deren Bezug zu negativen Globalisierungswirkungen in den USA im Gang - auch noch nach dem Abgang Donald Trumps im Januar 2021 (Benedikter 2021).

Operativ bedeutet Re-Globalisierung, dass Paradigmen der Nach-Kalter-KriegGlobalisierung zeit- und schichtenverschoben am Zusammenfluss von Beschleunigungs- und Verlangsamungskräften in Frage gestellt, verändert und möglicherweise auch voneinander isoliert dort erneuert werden, wo historische Konvergenzen das kontextuell begünstigen. Das zeigten zum Beispiel die Neuverhandlung des Nordamerikanischen Freihandelsabkommens NAFTA seit 2017 und seine Umwandlung in USMCA 2020; die Blockade, Nichtfortführung oder Aufkündigung internationaler Abkommen durch verschiedene Mächte, darunter die USA, zum Beispiel des Transpazifischen Partnerschaftsabkommens TPP und der Transatlantischen Handels- und Investitionspartnerschaft TTIP; sowie die tendenzielle Schwächung internationaler Institutionen wie der UN, der UNESCO, der WHO, der WTO oder des Internationalen Strafgerichtshofs in Den Haag (ICC) nicht zuletzt auch durch aufstrebende autoritäre Mächte. Die Schwächung sowohl von Geltungsansprüchen wie Handlungsfähigkeit von Globalisierungsinstitutionen betrifft letztlich auch das internationale Recht, das seit Jahren von vielen Staaten, darunter vom russischen Präsidenten in erklärt programmatischer Weise, gegenüber nationalen Gesetzgebungen und Interessen geschwächt wird (BBC 2020). Demgegenüber stehen Kräfte bisheriger globaler Ordnung, die die liberal-kosmopolitische Globalisierung retten wollen, indem sie einen Gegentrend setzen und neue Abkommen mit umso epochaleren Ansprüchen abschließen, wie etwa den „Globalen Pakt für Migration“ und den „Globalen Pakt zu Flüchtlingen“ der Internationalen Organisation für Migration (IOM) 2018 oder das EU-MERCOSUR-Abkommen 2019. Diese Abkommen dienen faktisch auch dazu, den stagnierenden Trend zu ,mehr Globalisierung“ zu behaupten und wiederzubeleben; ob sie diese damit auch besser kontextualisieren, wovon ihr Schicksal abhängen wird, bleibt abzuwarten.

Zusammenfassend bedeutet Re-Globalisierung als Untersuchungsgegenstand inter-, trans- und multidisziplinärer sozialwissenschaftlicher Analyse analog zu anderen historischen Umbruchphasen, dass die Kombination von Intentionen und Kapazitäten tektonische Bewegungen in Gang gebracht hat, die in oft widersprüchlichen Mustern zusammenwirken, aber auf bisherige Globalisierungslogiken mutmaßlich langfristig tiefenverändernd ausstrahlen. Dabei verstärkt sich bei vielen Bürger*innen unterschiedlicher politischer Systeme weitgehend übereinstimmend 
die Wahrnehmung, dass Expansionskräfte bisheriger Globalisierung nach und nach über ein verträgliches Maß hinausgegangen sind und eine zu große Öffnung ohne ausreichende Schutz- und Absicherungsmaßnahmen verwirklicht haben. So etwa bezogen auf Umweltzerstörung, Ressourcenverbrauch, ungeordnete Massenmigration oder Souveränitäts- und Subsidiaritätsverluste in verschiedenen Sektoren des Multiebenen-Regierens. Als Reaktion erlangen Rückzugs- und Schutzkräfte Zulauf - nun auch bei den Hauptarchitekten der bisherigen globalen Ordnung: den USA als militärischer, ökonomischer und politischer Sicherungsmacht und Europa als legalistischer Zivilmacht. Re-Globalisierung besteht in dieser Blickrichtung in der emotionalen Spaltung globalisierter Gesellschaften zwischen ihren Befürworter*innen und Gegner*innen. Das führt, ebenfalls systemübergreifend, zu einer Remoralisierung und damit teilweisen Re-Ideologisierung von Politik und Gesellschaft im Spannungsfeld zwischen Retro-, Anti- und Re-Globalisierung.

\section{Typologien der Re-Globalisierung}

Bei alledem scheinen sich nun einige typologische Grundmuster herauszubilden. Sie beziehen sich offenbar gegenläufig aufeinander und verstärken sich immer öfter zu Clustern. Unter diesen Grundmustern sind zunächst vor allem drei zu erwähnen.

Erstens scheint die Kontextualisierungsfrage sowohl bei Befürworter*innen wie Gegner*innen der Re-Globalisierung eine wesentliche Rolle zu spielen. Während in geschlossenen Systemen wie China Re-Globalisierungsbefürworter*innen auf demokratische Öffnung drängen und dazu Globalisierung mit liberalem Menschenrechtsimpetus stärken wollen (Hongkong), erfahren offene Systeme wie jene Europas und die angloamerikanische Welt im Gegenzug eine konservative Bewegung, die deren Offenheit mittlerweile als überzogen ansieht und Re-Globalisierung im Sinn von mehr (auch antizipativer) Grenzziehung interpretiert. So erhob es zum Beispiel der Sibiu-Gipfel der EU kurz vor den Wahlen des EU-Parlaments im Mai 2019 zur Vermeidung eines weiteren Rechtsrucks der EU-Wählerschaft erstmals zum Programm. Damit wurde den grundsätzlich seit 30 Jahren „öffnenden“ Grundstrategien der EU nach der Verlangsamung der Erweiterungsgeschwindigkeit erstmals auch offiziell entgegengewirkt und - zumindest rhetorisch - eine programmatische Trendwende eingeleitet, in deren Rahmen nun sogar die EU als der traditionell stärkste Propagator einer liberalen Weltordnung anstrebt, mittels Balance zwischen (alter) Grenzenlosigkeit und (neuen) Grenzziehungen zu einer positiveren Kontextualisierung der Globalisierung vorzustoßen. Dies bedeutet auch eine Diversifikation der Grundhaltung der EU bezüglich verschiedener Weltgegenden und politischer Systeme, wie etwa zu China und den USA. In dieses Gesamtbild passen auch neue globalpolitische Definitionen wie ,systemische Rivalität“ zu China und anderen Nicht-Demokratien oder neue Pläne wie der EU Green Deal der Kommission Ursula von der Leyen von 2019 bis 2020, der sich globalen Programmen eigenständig hinzustellt. Die stärkste Komponente dieser (zumindest rhetorischen) Trendwende ist die Kontextualisierung und „Glokalisierung“ europäischer Positionierung und grüner Erneuerung.

Eine zweite sich zusehends verdeutlichende Typologie ist die der gegenseitigen Vorwurfsargumentation zu fehlender politischer und ideologischer Balance. Konser- 
vative Lager, die auf Re-Globalisierung drängen, werfen Vertreter*innen bisheriger Globalisierungspraktiken internationaler Institutionen wie der UN oder der IOM eine überzogene Linksorientierung vor, die glaubte, sich des Neoliberalismus für ihre kosmopolitischen Ziele bedienen zu können und dabei scheiterte. Die führenden Internationalist*innen seien meist Linke, die sich - bewusst oder unbewusst - auf Elemente der 1968er-Emanzipationsbewegung beziehen, welche in den 1980er- und 1990er-Jahren zu einem idealistisch überzogenen Globalisierungsliberalismus ausinterpretiert wurde. Progressive Lager dagegen, welche die Grundelemente und -akteure bisheriger Globalisierung verteidigen, beschuldigen im Gegenzug konservative Re-Globalisierer*innen der regressiven und zuweilen sogar inhumanen Zerstörung einer ersten Menschheitsordnung, die durch die Nachkriegsordnung eingeleitet und seit 1990 zwar vielleicht zu rasch, aber doch in Kernelementen verwirklicht worden sei. Die Progressiven konzedieren in diesem Zusammenhang allerdings, dass sich ihre Hoffnung auf globale Demokratisierung als Trugschluss erwiesen hat.

Lagerübergreifend gibt es aber auch Zusammenschlüsse zwischen progressiven und konservativen Lagern. Gerade sie sind vielleicht besonders charakteristisch für den politischen „Mischcharakter“ der Re-Globalisierung. So haben sich in globalen Umweltbewegungen der Jugend wie Fridays for Future oder Extinction Rebellion (XR) Kräfte zusammengeschlossen, die im Prinzip ,post-postmodern“ und „,metamodern" konstituiert sind: Sie wollen über bisherige ideologische Gegensätze hinausgehen, da diese die Welt an den Rand des Abgrunds geführt hätten. Diese Jugendbewegungen beschuldigen beide ideologischen Lager: links wie rechts, die wahren Erneuerungsanforderungen bisheriger Globalisierung zu ignorieren und den Ernst der Lage nicht vollends zu erkennen. Ein „Weitermachen wie bisher“ schließen sie aus. Eine ,andere“, gemischt „progressiv-konservative“ Re-Globalisierung sei als Kooperationsanstrengung neuer Qualität systemübergreifend notwendig. Das würde eine koordinierte Erneuerung vieler bisheriger Elemente der Globalisierung, ihrer Institutionen und bisherigen Ideologien voraussetzen.

Das Augenmerk der Kritik dieser dritten, vielleicht am stärksten politisch aktiv "transzendierenden“ Re-Globalisierungstypologie richtet sich gegen den Abbau globaler Interaktion, zugleich aber auch gegen die Fortführung bisheriger Globalisierungsentgrenzungspraktiken. Diese Kritik zielt weniger gegen die bisherigen globalen Einrichtungen, sondern eher gegen jene Re-Globalisierer*innen regressiver Art, die sich als Kämpfer*innen gegen die „Übertreibungen“ der Globalisierung ausgeben, dabei aber letztlich selbst zerstörerische Politiken verfolgen - auch gegen die Welt der Natur. So etwa, um nur ein Beispiel zu nennen, gegen Brasiliens Präsident Jair Bolsonaro, der den Amazonas-Regenwald im Zeichen von Renationalisierung und Tribalisierung („Der Regenwald gehört nicht der Welt, sondern uns!“) sowie eines einseitig neoliberalen Wirtschafts- und Finanzverständnisses zerstören lässt:

Die Abholzung des Amazonas-Regenwaldes im Norden Brasiliens ist 2019 um $85 \%$ im Vergleich zum Jahr davor gestiegen, wie Daten belegen. 9.166 Quadratkilometer wurden gerodet - die höchste Zahl seit fünf Jahren, so das brasilianische Nationale Institut für Weltraumforschung. Im Jahr 2018 betrug die abgeholzte Fläche 4.946 Quadratkilometer. Die Daten wurden mit dem sa- 
tellitengestützten DETER-System gesammelt, das die Entwaldung in Echtzeit überwacht. Der drastische Anstieg ist das Ergebnis des ersten Amtsjahrs von Präsident Bolsonaro - er hatte die Rodungsauflagen stark gelockert (Austrian Press Agency 2020a).

Bolsonaro hatte die Abholzung des eigenen Regenwaldes ausdrücklich als Trotzmaßnahme gegen ,überzogene“ Globalisierung dargestellt, die sich moralisch und teilweise auch ökonomisch und politisch des brasilianischen Waldes zu bemächtigen suche, wie er anklagend ausgab.

\section{Beschleunigungs- versus Verlangsamungskräfte}

Bei alledem herrscht - trotz der gewissen gegenseitigen Annäherung in der Umweltfrage - bis auf weiteres ein Patt zwischen den verschiedenen Kraftblöcken. Beschleunigungskräfte ,klassischer“ Globalisierung kommen vor allem aus Technologie und Wirtschaft; Verlangsamungs-, Eindämmungs- und Gegenkräfte aus Populismus, neuen politischen Tribalismen, Re-Nationalisierung und der Rückkehr der Geographie als Einflussfaktor; transideologische Korrekturkräfte aus Jugendbewegungen, die wachsende Aufmerksamkeit für Kontextpolitiken, eine neue Zuwendung zur Wissenschaft sowie zum öffentlichen Imaginären (Imaginationspolitik) entwickeln. Für das Lager transideologischer Korrekturkräfte wird die Eigenbewertung (und -bearbeitung) der öffentlichen Imagination sogar zum politischen und ethischen Imperativ (Faessel et al. 2019). Denn sie erkennen in ihr das entscheidende, eigentliche „Schlachtfeld“ der Re-Globalisierung, auf dem sich deren Richtungnahme (links, rechts oder beides zugleich) entscheiden wird. Beschleunigungskräfte bestehen aber auch in der interessensgeleiteten Vereinnahmung der Globalisierung durch parastaatliche Technologie- und Wirtschaftskräfte nicht mehr nur des Westens (Silicon Valley), sondern inzwischen auch Chinas, Russlands und des Fernen Ostens. Verlangsamungs- und Entglobalisierungskräfte reagieren darauf und betonen im Gegenzug Lokalität, Autonomie und Selbstsuffizienz. Transideologische Korrekturkräfte schließlich drängen auf eine ,glokalere“ Globalisierung, die das Augenmerk auf neue Balancen legt und dazu auch ihre Institutionen neu aufstellt.

\section{Der Umbruch von der Globalisierung in die Re-Globalisierung erfolgt (mindestens) sechsdimensional}

Zugleich verschärft sich die Profilierung „konkurrierender Modernitätskonzepte“ zwischen unterschiedlichen Leitkulturen, die manche mit verschiedenen Zivilisationen gleichsetzen. Unter den Treibern dieser Entwicklung zu Diversifizierung und Pluralität sind China, die islamische Welt, Europa und die USA. Ihre fortgesetzten kulturellen Differenzen, die sich mit der globalen liberalen Ordnung entgegen der hohen Hoffnungen und trotz technologischer Nivellierung kaum verringert haben, tragen seit Jahren zum Umbruch in die Re-Globalisierungsepoche bei. Nur ein Beispiel unter vielen ist die Niederlage des Westens in Afghanistan mit dem unpro- 
fessionellen Abzug 2021 und der sofortigen Rückkehr der Taliban. Trotz 20 Jahren relativ erfolgreicher emanzipativer Bestrebungen in den urbanen Zentren des Landes ist es dem Westen nicht gelungen, eine Mehrheit zur Annahme von Modernitäts- und Demokratiewerten zu bewegen. Der Grund dafür ist unter anderem eine Kultur, die sich - tief verwurzelt und in einem Stadt-Land-Gegensatz verschanzt - der Demokratisierung widersetzt, in diesem Fall eine sehr konservative Auslegung des Islam. Die Rückkehr der Religion im Kern der Zivilisationen unterstützt die Schärfung kultureller Differenzen und Andersheiten innerhalb der globalen Ordnung. Obwohl sich der ,postmoderne“ westliche Säkularismus universal glaubte und einen humanistisch verfeinerten Menschheitsanspruch erhob, den er nach dem Ende des Kalten Krieges mittels Globalisierung endgültig im Sinn eines „Endes der Geschichte“ (Francis Fukuyama) durchsetzen wollte, war er in Wirklichkeit nie allgemein anerkannt oder auch nur passiv akzeptiert. Das Beispiel Afghanistan zeigt, dass Kultur und Religion in der Re-Globalisierungsphase zur Herausarbeitung von Unvereinbarkeiten beitragen, die jenseits dialogischer Differenzen liegen und die Welt in prinzipiell inkompatible Wertevorstellungen gliedern. Das entkräftet bisherige globale Integrationsideologien und schafft zugleich neue Asymmetrien. Für viele hat der „Kampf der Zivilisationen“ (Huntington 1996) erst seit der Re-Globalisierungsphase wirklich begonnen. Andere dagegen sehen Re-Globalisierung selbst als makrokulturell begründete Ideologie an, die genau diesen Kampf nicht in gemeinsame neue Bahnen lenken will, sondern durch ihre Konzepte erst auf breiter Front miterzeugt.

Wichtig ist angesichts solcher Synchronie widerstrebender Kräfte jedenfalls, ein gemeinsames Motiv zu sehen: Der Umbruch von der Globalisierung in die ReGlobalisierung erfolgt nicht nur sektoral oder disziplinär. Er erfolgt in allen sechs Dimensionen ausdifferenzierter, (post-post-)moderner und im Gefolge von mehr oder weniger drei Jahrzehnten Globalisierung (1990-2020) transnational konstituierter Gesellschaften: 1) Wirtschaft (Herausforderung des Neoliberalismus durch eine Kreislauf- und Post-Wachstumsökonomie und eine globale „grüne Wende“, die indirekt von wiederkehrenden anti-neoliberalen Ideen von Nationalwirtschaften paradoxal unterstützt wird, so etwa von Donald Trumps „America First“); 2) Politik (vom Washington Consensus zur Multipolarität); 3) Kultur (vom westlich-internationalistischen Multikulturalismus zur Renaissance nationaler Kulturen als Leitkulturen); 4) Religion (von der Säkularisierung zur Renaissance der Religionen inzwischen auch in laizistischen Gesellschaften); 5) Demographie (von Ein-Kind-Politik und Bevölkerungskontrolle zum ,demographischen Imperialismus“, der mit Bevölkerungs- und Generationsverschiebungen Außenpolitik macht und Wirtschaft lenkt, so wie etwa in der nationalistischen politischen Agitation Recep Tyyip Erdogans gegenüber Auslandstürk*innen); 6) Technologie (von Digitalisierung und Mensch-Maschine-Interaktion zu Algokratie und Mensch-Maschine-Konvergenz). Dazu kommt, als Meta-Treiber, der alle diese sechs Dimensionen überwölbt, informiert und zum Teil auch ideologisiert, die Zuspitzung der Umweltfrage: vom Nischenthema zum Sammelbecken von Anti-Politiken gegen bisherige Globalisierungspraktiken. 


\section{Perspektiven der Re-Globalisierung}

Alle Lager, die in der Re-Globalisierung heute bezogen auf bisherige Globalisierungslogiken im Spiel sind, die Kontinuitäts-, die Gegen- und die Korrekturkräfte, bilden keine klaren Einheiten, sondern sind in sich heterogen konstituiert. Alle weisen linke und rechte Flügel auf, die sich nach innen bekämpfen, um nach aussen Gestaltungsvorrang zu erlangen. Ihre Normen und Werte könnten nicht gespaltener sein. Und sie könnten sich nicht schneller in ihren Konstellationen verändern - was ein ganzheitliches Gestalten von Re-Globalisierung erschwert, weil sich diese nicht als eine zumindest temporär stabile, sondern höchst dynamische Situation erweist. Dabei gilt es zu beachten, dass „Re-Globalisierung“ nicht einfach mit „,neuen Herausforderungen an die Globalisierung“ deckungsgleich ist, zu denen sie gerade in der deutschsprachigen Diskussion oft abgeschwächt wird. Stattdessen stellt sie ein Thema dar, das auf keinen seiner Teilbereiche reduzierbar ist. Um Re-Globalisierung in den kommenden Jahren nicht nur passiv zu erfahren, sondern aktiv zu gestalten, sind im Wesentlichen fünf zentrale Perspektiven zu beachten: Die Globalisierung muss 1) korrigiert und verfeinert (refining), 2) neu kontextualisiert, gerahmt und angepasst (reframing), 3) reformiert (reforming), 4) neu definiert (redefining), und 5) mit einer neuen Vision versehen werden (revisioning) (Benedikter und Kofler 2019).

Re-Globalisierung nimmt am Überschneidungspunkt dieser fünf grundlegenden Perspektiven eine krisenhafte Transformationsfunktion bisheriger Globalisierungsideen und -paradigmen ein. Das löst einen komplex verketteten Prozess der Instabilitäten aus, der die nächsten Jahre bestimmen wird, und zwar voraussichtlich sowohl im Ganzen wie in Einzelsymptomen und -ereignissen.

\section{Re-Globalisierung und Imaginäres}

Ein nicht zu unterschätzender Aspekt bei alledem ist die Veränderung des öffentlichen Imaginären und, allgemeiner ausgedrückt, der Vorstellungen darüber, was Globalisierung ist, was sie bewirkt, was sie sein soll, und wohin sie zielt. Diese Vorstellungen haben sich in den vergangenen Jahren stark verändert. Sie nehmen heute sowohl populäre wie wissenschaftliche Formen an. Wenn Manfred Steger 2008 mit großer Hoffnung vom „Entstehen eines globalen Imaginären“ sprach, so gilt 2021, dass inzwischen die Gegenbewegung, die kritische Dekonstruktion dieses - angeblich progressiven, oft aber im Dienst von Interessen gebrauchten - Imaginären vor sich geht (Steger 2008). Kritikpunkte sind unter anderem die idealistische Abstraktion dieses ,globalen Imaginären“, das die Imagination eines ,guten Globalismus“ ohne ausreichend realistischen Unterbau war; sein Wunsch- und Begehrenscharakter und seine - oft sogar programmatische - Entterritorialisierung, die auch zur (rhetorischen, theoretischen und politischen) Delokalisierung und De-Historisierung von Globalisierung führte. Letzteres ist auch Gegenstand der systemübergreifend wachsenden Kritik an der ,Elite-Globalisierung“. Sie könnte, unabhängig von Verschwörungstheorien, welche in den sozialen Medien massenhaft als Trittbrettfahrer auf sie aufspringen, eines der großen Themen der kommenden Jahre werden. 
In der Tat hat Sozialwissenschaft gerade erst begonnen, Re-Globalisierung in ihren hermeneutischen Bezügen zwischen Teilen und Ganzheiten, Gestaltern und Gestaltetem zu verstehen - auch bezogen auf das diese je unterschiedlich durchdringende Imaginäre (Mazlish 2013). Die Frage ist dabei einerseits, ob - wie manche behaupten - nach drei Jahrzehnten nun auch imaginär „Globalisierung zu Ende geht“ (Klude 2019). Andererseits, ob es seit den 1990er-Jahren überhaupt eine ,globale Globalisierung“ (Berger 2002) gegeben hat, oder ob neoliberal-kosmopolitische Globalisierungsvorstellungen und die von ihnen mehr oder weniger bewusst konstruierten Idealbilder einer offenen, durchlässigen und freien Welt nicht vielmehr die Tatsache verschleierten, dass Globalisierung nie global war. Sondern dass sie eher in einer Mentalität des ,Sowohl-als-auch“, also einer Koexistenz von Globalisierung in bestimmten Gebieten mit Nicht-Globalisierung in anderen, stehen blieb (Zukunftsinstitut o.J.). Indifferenz wurde dabei vom kosmopolitischen Neoliberalismus als Tolerierung von Unterschieden verkauft. Demgegenüber ist die heute weitgehend lagerübergreifend - vorgebrachte Forderung nach einer ,neuen Globalisierung“ eine, die die positiven Globalisierungsaspekte behalten, ihre negativen Begleiterscheinungen beseitigen und dabei eine neue Mitte zwischen hoch- und unterentwickelter Globalisierung als Leitkonzept entwickeln will. Eben in diese ,neue Globalisierung“, die sich auf konkrete Territorien, Klassen, Machtverhältnisse und Bevölkerungen bezieht, könnte eine konstruktive und kooperative Interpretation von „Re-Globalisierung“ einmünden (Zukunftsinstitut o.J.).

\section{Gegensätze der Perspektivierung}

Aber nicht nur die neoliberal-kosmopolitische Globalisierung wird derzeit zurückgesetzt. Sondern auch der „Globalismus“ als Ideologie und Denkweise wird durch Re-Globalisierung sowohl faktisch wie theoretisch hinterfragt. Re-Globalisierung beschreibt einen umfassenden Prozess, der wahrscheinlich nicht bald enden wird, und in dem sowohl ideologisch progressive als auch regressive Kräfte im Spiel sind, die sich gegenseitig bekämpfen. Wirtschaft, Politik, Kultur, Religion, Technologie und Demografie: Alle Bereiche sind gleichzeitig betroffen und werden oft gegeneinander ausgespielt. Was die unterschiedlichen Fraktionen eint, ist die Frage: Wie können die Turbulenzen der Re-Globalisierung in eine produktive Erneuerung jener Globalisierung und jenes Globalismus einmünden, wie wir sie in ihrer überwiegend neoliberal-kosmopolitischen Konzeption der vergangenen Jahrzehnte kannten?

Mit Blick auf diese Szenerie sind sozialpsychologisch vor allem Freiheiten der Meinungsbildung zur Perspektivierung wichtig. Doch wie eine Umfrage des Instituts für Demoskopie Allensbach vom Juni 2021 ergab, trauten sich $202155 \%$ der Deutschen nicht mehr, ihre Meinung offen zu sagen, da sie Nachteile für ihr Privatleben befürchteten. Das war das schlechteste Ergebnis in Bezug auf Redeund Meinungsfreiheit in der Geschichte der Umfrage, nämlich seit 1953. In anderen demokratischen Gesellschaften Europas dürfte es ähnlich aussehen. Politische Korrektheit lähmt offene Gesellschaften, ihr volles Potential zu aktivieren, wie es Demokratie vorsieht - während autoritäre Gesellschaften die Zügel weiter anziehen. Auch das ist ein Teil der Re-Globalisierungskonstellation. Wird dieser Trend nicht 
umgekehrt, kann auch die Re-Globalisierungsdebatte nicht ihre vollen positiven Effekte zur Erzeugung von Perspektiven entfalten.

Ein (produktiv) ambivalenter Faktor in der Perspektivierung ist die Repolitisierung insbesondere der Jugend. $\mathrm{Zu}$ ihr hat der zivilgesellschaftliche Sektor, einschließlich die Aktivitäten demokratisch nicht kontrollierter Akteure wie privater NGOs, mittels drängender Globalisierungsfragen wie die der Migration beigetragen. Mutmaßlich auch als Reaktion auf Globalisierungswirkungen sind einige der für ihre bisherige Gestalt wichtigsten Persönlichkeiten inzwischen von ihren bisherigen Leitfunktionen zurückgetreten, wie etwa die Google-Gründer Larry Page und Sergey Brin im Jahr 2019, mit unklaren Folgen. Der Rückzug der Gründergeneration der „Ultra“-Globalisierung könnte das Ende einer Epoche ankündigen, die letztlich kürzer war als angenommen. Sie könnte aber auch den Weg für einen noch stärkeren Einfluss von Technikriesen jenseits Demokratie- versus Nicht-DemokratieDichotomien ebnen.

\section{Zwölf offene Baustellen}

In der westlichen Hemisphäre führt die Polarisierung über Re-Globalisierungsfragen inzwischen - und das ist einer ihrer vorläufig wichtigsten Effekte - zu einer Radikalisierung und zum Schrumpfen der für die Demokratie entscheidenden Mitte. So beispielsweise in den USA, wo Barack Obama seit 2019 nicht nur den Verlust von Faktizitäten beklagte, die die unverzichtbare Objektivitätsbasis für demokratischen Dialog darstelle, sondern im November 2019 die Demokratische Partei auch davor warnte, für gemäßigte Wähler in der Mitte ,unwählbar“ zu werden. Die Progressiven seien als Reaktion auf einen zu weit nach rechts gerückten Präsidenten Donald Trump selbst zu weit nach links gerückt. Die Folge solchen Aus-der-Mitte-Driftens ist ein Verfall der demokratischen Kultur, der den Aufstieg autoritärer Regime wie China mit seinem „Neue Seidenstraße“-Projekt indirekt fördert.

In dieser Situation doppelter Spaltung sowohl innerhalb offener Gesellschaften wie zwischen offenen und autoritären Gesellschaften, die UN-Generalsekretär António Guterres als ,Zeitalter des Misstrauens“ bezeichnete, gibt es zwölf Bereiche, in denen Muster neu geordnet werden können - und auf die man sich möglicherweise stützen kann, um die Globalisierung zu überdenken, neu zu konstruieren und aufzubauen. Diese zwölf Bereiche oder Handlungsfelder sind:

- Multipolarität (Entwicklung neuer Strategien für den Umgang mit der Vervielfältigung verschiedenartiger globaler Ordnungsvorstellungen und Ordnungen);

- Maßstab (konstruktiv mit Asymmetrien arbeiten und zum Beispiel die Frage stellen, ob groß immer schön ist, etwa die Größe der wichtigsten politischen Mächte oder der globalen Internetgiganten im Vergleich zu kleineren und begrenzteren Gemeinschaften);

- Gleichgewicht (zwischen Systemen, Zahlen und Akteuren);

- Kontext (einschließlich Glokalisierung);

- Grenze (Arbeit mit dem „Seneca-Effekt“ maximaler Sättigung und Kipppunktberechnungen, um zu verhindern, dass Hochrisikosektoren überschießen); 
- Multi-Resilienz als Transformationsdesign (Stärkung und Vernetzung von Resilienzstrategien bei Vermittlung zwischen unterschiedlichen Größen und Wertesystemen);

- Zukünftebildung (Futures literacy, das heißt Stärkung und Verknüpfung interaktiver Vorhersage-, Vorausschau- und Antizipationsansätze als Breitenfähigkeit, die zum transversalen Schulfach werden könnte);

- Inter- und Transdisziplinarität (Vernetzung und Überblick über das ,große Ganze“ durch Berücksichtigung der gegenseitigen Wechselwirkungen zwischen den sechs Dimensionen wirtschaftlicher, politischer, kultureller, religiöser, technologischer und demografischer Re-Globalisierungs-Logiken);

- Nachhaltigkeit (Anwendung einer rückgekoppelten Langzeitperspektive);

- Narrative Literacy / Narrative Balancing (Zurückdrängen politischer Korrektheit im akademischen und öffentlichen Diskurs, um Raum für unterschiedliche Darstellungen des Geschehens und seiner Interpretation zu schaffen);

- Imaginationspolitiken und -ökonomien (Berücksichtigung des zunehmenden Einflusses immaterieller Faktoren und der Mehrdeutigkeit von Information auf alle Aspekte digitalisierter Gesellschaften);

- Gleichheit und Gewissheit (systemübergreifende Bekämpfung (countering) von Ungleichheit und Unsicherheit).

Um diese Ziele zu integrieren, sollten innovative politische Strategien und strategische Maßnahmen über die Vereinten Nationen und andere globale Gremien in Gang gesetzt werden, um mit allen interessierten Akteuren auf möglichst einbeziehende Weise zusammenzuarbeiten.

\section{Ausblick}

Angesichts der aufgewiesenen Grundlagenaspekte lautet die Frage nicht „Gibt es die Re-Globalisierung?“, sondern „Wohin geht die Re-Globalisierung? Und was kann Re-Globalisierung bewirken?" Diese Frage wurde seit dem Beginn der NachKalter-Krieg-Globalisierungsphase am Beginn der 1990er-Jahre zu selten gestellt - vor allem im deutschen Sprachraum, der sich ab dem Fall der Berliner Mauer zum Ost-West-Vermittler und Exportweltmeister entwickelte und sich (auch) damit wie die demokratische Leitmacht USA überproportional abhängig von Globalisierungsmechanismen machte. Aufbruch und Euphorie prägten in der Folge über zwei Jahrzehnte lang eine fast durchgängig positive Konnotation von Globalisierung. Die (instrumentelle) Allianz von neoliberal-profitorientierten und kosmopolitisch-progressiven Kräften handhabte den Globalisierungsbegriff weitgehend unkritisch und zum Teil hart an der Grenze zur Propaganda. Seit einigen Jahren schlägt das Pendel jedoch zu mehr Skepsis um, die auf vielen Gebieten in Abwehr- und Gegenaktivitäten mündet. Wie muss die Globalisierung nun, nach einer Inkubationsphase von immerhin 30 Jahren, reformiert werden, um Minimalziele der Weltgemeinschaft wie die 17 SDGs (Sustainable Development Goals) im Rahmen der UN-Agenda $2030 \mathrm{zu}$ erreichen? Diese Fragen sollten von den Sozialwissenschaften im deutschen Sprachraum mit mehr Mut zur Ganzheitlichkeit als bisher gestellt werden. Die Geschichte 
des 20. Jahrhunderts mit ihren totalitären Ansprüchen und katastrophalen Ergebnissen hat im deutschsprachigen Wissenschaftsbereich auch eine irrationale Angst vor auch nur im Ansatz ,ganzheitlichen“ Begriffen wie Zukunft, Globalisierung, Globalismus oder ,ganze Welt" verursacht - was darauf hindeutet, dass die Vergangenheit der ersten Hälfte des 20. Jahrhunderts zwar in nie dagewesener Weise intellektuell aufgenommen, aber noch lange nicht verarbeitet wurde.

Die Frage nach der wünschenswerten Zukunft der Globalisierung in Form einer „Re-Globalisierung“ sollte jedoch auch im deutschen Sprachraum verstärkt gestellt werden. Denn neben positiven werden auch negative Globalisierungswirkungen faktisch immer wichtiger. Dazu stehen in den kommenden Jahren zum Teil wohl irreversible Richtungsentscheidungen an: in der Umweltkrise, der Energiefrage, der Einheit Europas und des Westens und der Reform und zeitgemäßen Erneuerung globaler Institutionen. Manche dieser Entscheidungen werden auf internationaler Ebene kaum, nur vorübergehend oder nur oberflächlich konsensual zu treffen sein und trotzdem weitreichende Folgen auch für lokale Gemeinschaften über Jahrzehnte zeitigen. Zugleich sind ausnahmslos alle globalen Institutionen und Einrichtungen, sowohl internationaler (UN) wie privater (NGOs) Art im Übergang oder in der Krise. Das zeigt einen erhöhten Reflexionsbedarf an, den sie allein nicht bewältigen können. Wissenschaft und Zivilgesellschaft sollten dabei helfen.

Nicht nur die Situation der WTO, sondern auch der Weltbank oder des Internationalen Währungsfonds sowie anderer transnationaler Einrichtungen wie etwa des Olympischen Komitees, des Weltfußballverbandes FIFA oder der Welttourismusorganisation UNWTO - mit Versuchen unter anderem Chinas, diese Institutionen durch eigene Parallelgründungen zu relativieren oder gar zu ersetzen - zeigt: Heute besteht offenbar eine Nadelöhrsituation der Globalisierung, in der sich ihre Zukunft zwischen Kooperation und Konkurrenz, zwischen alten und neuen Zusammenschlüssen und Autonomisierungsprozessen entscheidet. Das hat auch mit der Konvergenz von Problemen zu tun, die lokal, regional und national allein nicht mehr zu lösen sind - und deshalb im Gegenzug gegen (scheinbare) Fremdbestimmung neue nationale Alleingänge und Populismen hervorrufen, die sich zum Teil auf wiederkehrende ethnische und soziale Tribalismen stützen. Doch die Erfahrung der letzten Jahre hat auch gezeigt: Nationalstaaten sind zur Lösung globaler Probleme zu klein, zur Lösung lokaler Probleme zu groß. Kontextualisierung, Maßstab (scale) und Glokalität sind deshalb die Zukunft der Globalisierung - nicht die Wiederkehr egoistischer Logiken, die letztlich nur zu neuen geopolitischen „Schachspielen“ und Konflikten führen wird.

Tatsache bleibt: Weltweit wirken immer mehr immer fundamentalere Probleme zusammen und verstärken sich gegenseitig. Dabei geraten bisherige Globalisierungsverständnisse, -strategien und -institutionen deutlicher als vordem an ihre Grenzen. In Situationen komprimierter Problemsynchronien entsteht Überforderungsdruck und deshalb Streit und Spaltung - was die Sozialwissenschaften die „Kontrollverlust-Psychologie“ nennen. Zugleich häufen sich die Zeichen sowohl für einen Bruch wie eine Regression bisheriger Globalisierungsordnungen, -pratiken und -habitus. Das macht zusammengenommen grundlegende Reformen bisheriger Globalisierung nötig - worin die Mehrheit der Beobachter*innen inzwischen über- 
einstimmt. Worin sie nicht übereinstimmt, sind das „Wie“, die genauen Methoden dazu, die Stoßrichtung und der anzupeilende Zielraum.

Wir fassen demnach die aktuell auftretende Gleichzeitigkeit von teilweise irreversiblen Brüchen, Regressionen, Überziehungssymptomen (overshoot phenomena) und Reformnotwendigkeiten von Globalisierung in ihren relevanten Sektoren im Sammelbegriff „Re-Globalisierung“ zusammen (Benedikter 2021). „Re-Globalisierung" kann im Angesicht dieser Entwicklungen als experimenteller Epochenbegriff dienen. Wie lange, ist offen. In welchem Umfang, bleibt der Diskussion überlassen.

Fazit? Obwohl sich sowohl Erkenntnisgegenstand wie auch Begriffsklärung von „Re-Globalisierung“ erst am Beginn befinden, ist zumindest einiges wenige klar. Erstens: „Re-Globalisierung“ ist (noch) kein normativer Begriff, kein genau umrissener kognitiver Gegenstand und auch keine Methode. In erster Linie ist sie ein multidimensionaler, pluriperspektivisch offener sozioökonomischer und politischer Prozess, also ein zunächst deskriptiv zu umkreisender Beobachtungsgegenstand. Dementsprechend ist „Re-Globalisierung“ eine unmissverständlich erst im Werden begriffene Epochenkennzeichnung. Und: Re-Globalisierung ist sowohl ein passiver wie ein aktiver Vorgang. Passiv verstanden, ist „Re-Globalisierung“ ein Schlagwort, um zentrale Aspekte des „,Transformationsdesigns“ der Gegenwart zu umreißen. Die Herausforderung der aktiven Gestaltung von „Re-Globalisierung“ dagegen wird die Politik- und Gesellschaftsstrategien der kommenden Jahre prägen - willentlich oder unwillentlich, und sowohl in den Regionen Europas und des Westens wie international. Was genau also wird einerseits mit der Epochenkennzeichnung Re-Globalisierung und was andererseits mit der Herausforderung Re-Globalisierung geschehen? Und: Wie können wir ihre sehr verschiedenartigen Aspekte zusammenführen, um sie ganzheitlicher als bisher zu gestalten? Das werden die kommenden Jahre zeigen. Dazu wird von der sozialwissenschaftlichen Forschung mehr - und noch viel mehr Spezifisches als im hier skizzierten Umriss - zu sagen sein.

\section{Literatur}

Austrian Press Agency (2020a, 14. Jan.). Amazonas-Abholzung um 85\% gestiegen. news.orf.at. https:// orf.at/stories/3150993/. Zugegriffen: 22. Nov. 2021.

Austrian Press Agency (2020b, 25. Juni). Guterres für Multilateralismus. news.orf.at. https://orf.at/stories/ 3171145/. Zugegriffen: 22. Nov. 2021.

BBC (2020, 15. Jan.). Russian government resigns as Vladimir Putin plans future. https://www.bbc.com/ news/world-europe-51120166. Zugegriffen: 17. Aug. 2021.

Benedikter, R. (2012). USA: Abkehr von Europa? Obama gegen Romney: Hintergründe und Perspektiven für Europa bis 2016. Hannover: Heinz Heise.

Benedikter, R. (2021). What is re-globalization? New Global Studies, 15(1), 73-84.

Benedikter, R., \& Kofler, I. (2019). Globalization's current transition phase: The 5 R's. Global-e. A Global Studies Journal. https://globalejournal.org/global-e/august-2019/globalizations-current-transitionphase-5-rs. Zugegriffen: 17. Aug. 2021.

Berger, R. (2002, 6. März). Globale Globalisierung. Die Welt. https://www.welt.de/print-welt/article377727/ Globale-Globalisierung.html. Zugegriffen: 17. Aug. 2021.

El-Safadi, M. (2020). „Weltordnung ohne Hüter“. Ein Gespräch mit dem Politologen Prof. Dr. Herfried Münkler. Welt Trends - Das Außenpolitische Journal, 163(4), 24-28.

Faessel, V., Falk, R., \& Curtin, M. (Hrsg.). (2019). On public imagination. A political and ethical imperative. London: Routledge. 
Guterres, A. (2020). Remarks to the general assembly on the secretary-general priorities for 2020. United Nations. https://www.un.org/sg/en/content/sg/speeches/2020-01-22/remarks-general-assemblypriorities-for-2020. Zugegriffen: 17. Aug. 2021.

Henriques, G. (2020, 17. Apr.). What is metamodernism? Metamodernism is the cultural code that comes after postmodernism. Psychology Today. https:/www.psychologytoday.com/us/blog/theoryknowledge/202004/what-is-metamodernism. Zugegriffen: 22. Nov. 2021.

Huntington, S. P. (1996). Kampf der Kulturen. Die Neugestaltung der Weltpolitik im 21. Jahrhundert. München: Europa-Verlag.

Klude, C. (2019, 25. Juni). Das Ende der Globalisierung. Capital. https://www.capital.de/wirtschaftpolitik/das-ende-der-globalisierung. Zugegriffen: 17. Aug. 2021.

Mazlish, B. (2013). Part and wholes. The seven-dimensional approach of Roland Benedikter to the analysis of globalization - and its predeccesors in the history of the interdisciplinary social sciences. An affirmative reading. Transcience. https://www2.hu-berlin.de/transcience/Vol4_Issue1_2013_36_ 39.pdf. Zugegriffen: 17. Aug. 2021.

Miller, R. (2019). Transforming the future. Anticipation in the 21st century. London: Routledge.

Mishra, P. (2020). Grand Illusions. It's time to abandon the intellectual narcissism of cold war Western liberalism. The New York Review of Books. https://www.nybooks.com/articles/2020/11/19/liberalismgrand-illusions/. Zugegriffen: 17. Aug. 2021.

Müller, H. (2019, 13. Okt.). Unruhe ist die neue Normalität. Der Spiegel. https://m.spiegel.de/wirtschaft/ soziales/globale-konjunktur-unruhe-ist-die-neue-normalitaet-a-1291328.html. Zugegriffen: 17. Aug. 2021.

Nealon, J. (2012). Post-modernism. Or the cultural logic of just-in-time capitalism. Stanford: Stanford University Press.

Richards, W. (2020, 29. Okt.). Nick Cave discusses receiving criticism online: "Negativity, cynicism and resentment will not do". New Musical Express. https://www.nme.com/news/music/nick-cavediscusses-receiving-criticism-online-negativity-cynicism-and-resentment-will-not-do-2803413? utm_source=rss\&utm_medium=rss\&utm_campaign=nick-cave-discusses-receiving-criticism-onlinenegativity-cynicism-and-resentment-will-not-do. Zugegriffen: 17. Aug. 2021.

Steger, M. (2001). Globalism: The new market ideology. Lanham: Rowman \& Littlefield.

Steger, M. (2008). The rise of the global imaginary. Oxford: Oxford University Press.

Steger, M., Benedikter, R., \& Pechlaner, H. (Hrsg.). (2023). Globalization: past, present, future. Berkeley: University of California Press.

Turner, L. (2015, 12. Jan.). Metamodernism: A brief introduction. Notes on metamodernism. http://www. metamodernism.com/2015/01/12/metamodernism-a-brief-introduction/. Zugegriffen: 22. Nov. 2021.

Zukunftsinstitut (o. J.). Die Potenziale der Globalisierung. https://www.zukunftsinstitut.de/artikel/diepotenziale-der-globalisierung/https. Zugegriffen: 17. Aug. 2021. 